SECTION 4. Computer science, computer engineering and automation.

Denis Sergeevich Solovjev Assistant of the department «Information systems and information security» Tambov State Technical University, Russia solovjevdenis@mail.ru

\title{
SOFTWARE FOR VISUALIZATION OF THICKNESS ELECTROPLATED COVERING DISTRIBUTION OVER THE SURFACE OF DETAILS
}

Abstract: The paper presents the problem of visualizing of thickness electroplated covering distribution over the surface of details. The methods of geometric modeling to solve the problem are describes. Visualization of thickness covering distribution for the specific example received in the software developed by the author are demonstrates.

Keywords: software, thickness of electroplating covering, 3D model of object.

Citation: Solovjev DS (2014) SOFTWARE FOR VISUALIZATION OF THICKNESS ELECTROPLATED COVERING DISTRIBUTION OVER THE SURFACE OF DETAILS. ISJ Theoretical \& Applied Science 9 (17): 28-30. doi: http://dx.doi.org/10.15863/TAS.2014.09.17.4

\section{УДК 621.35}

\section{ПРОГРАММНОЕ ОБЕСПЕЧЕНИЕ ДЛЯ ВИЗУАЛИЗАЦИИ РАСПРЕДЕЛЕНИЯ ТОЛЩИНЫ ГАЛЬВАНИЧЕСКОГО ПОКРЫТИЯ ПО ПОВЕРХНОСТИ ДЕТАЛИ}

\begin{abstract}
Аннотация: В работе приводится проблема визуализации распределения толщиньы гальванического покрытия по поверхности детали. Описываются методы геометрического моделирования для решения поставленной проблемы. Приводится визуализация распределения толщины покрытия для конкретного примера посредством разработанного автором программного обеспечения.
\end{abstract}

Ключевые слова: программное обеспечение, толщина гальванического покрытия, 3D модель объекта.

В работах [1, с. 71; 2, с. 128] описывается принцип перенесения растровой развертки цифрового изображения из области телевизионного вещания в технологию гальванической обработки изделий, сущность которого состоит в том, что для управления гальваническим осаждением металла используется развертка, а градация напряжения на анодных секциях осуществляется при движении по строкам в соответствующие промежутки времени. Для предлагаемого процесса разработана система управления, позволяющая отыскивать оптимальный режим функционирования анодных секций [3, с. 15]. Одним из результатов численного решения задачи оптимального управления таким гальваническим процессом является распределение толщины покрытия по поверхности детали, для визуализации которого автором разработано соответствующее программное обеспечение [4]. Входными данными программного обеспечения для визуализации распределения толщины покрытия будет следующий набор координат:

$$
\left\{\mathrm{x}_{1}, \mathrm{y}_{1}, \mathrm{z}_{1}, \delta_{1}\right\}, . .,\left\{\mathrm{x}_{\mathrm{k}}, \mathrm{y}_{\mathrm{k}}, \mathrm{z}_{\mathrm{k}}, \delta_{\mathrm{k}}\right\}, . .,\left\{\mathrm{x}_{\mathrm{K}}, \mathrm{y}_{\mathrm{K}}, \mathrm{z}_{\mathrm{K}}, \delta_{\mathrm{K}}\right\}
$$

где $\mathrm{K}$ - число узловых точек, принадлежащих поверхности детали; $\mathrm{xk}, \mathrm{yk}_{\mathrm{k}}, \mathrm{Z}_{\mathrm{k}}, \delta_{\mathrm{k}}$ пространственные координаты k-ой узловой точки и толщина покрытия в ней.

Точка с k-м индексом объекта, описывающего деталь, имеет 3 пространственных и 1 количественную характеристики. Для визуализации толщины покрытия в k-ой точке используются цветовые градации. Традиционно для моделирования трехмерных объектов используются треугольники, в нашем случае, с цветами вершин, полученными в результате 
цветовой обработки массива исходных данных. При этом все необходимые для построения проекции и установления нужного ракурса преобразования координат описываются матрицами размером 4x4 и представляются в виде суперпозиции некоторых основных преобразований: переноса точки в пространстве на фиксированный вектор, поворота вокруг указанной оси на заданный угол, масштабирования вдоль какой-либо оси, сдвига, перспективы и проецирования [5]. Для построения правильного изображения трехмерных объектов необходимо уметь определять, какие части объекта будут видны при заданном положении, а какие будут закрыты другими гранями объекта. В качестве возможного решения поставленной задачи предлагается использование алгоритма Z-буфера. Возможно, дополнительно понадобится визуализация рельефа распределения толщины покрытия на заданном фрагменте объекта. Для реализации рельефа фрагмента предлагается воспользоваться поверхностью Безье, использующейся при кусочном моделировании для задания и изменения формы куска, представляющего собой пространственную решетку из сплайнов контрольных точек [6].

Рассмотрим пример. Пусть найдено решение задачи оптимального управления нанесением никелевого покрытия на деталь (гайка для автоматических ворот), изображенную на рис. 1а. Тогда на рис. 16 цветом продемонстрировано распределение толщины покрытия по поверхности детали-катода, полученное в результате численного расчета. Программа позволяет задавать различные граничные цвета, указывать количество их градаций, а также осуществлять все основные трехмерные преобразования. Программное обеспечение также способно осуществлять визуализацию рельефа распределения толщины покрытия на интересующем проектировщика фрагменте поверхности объекта (рис. 1в).

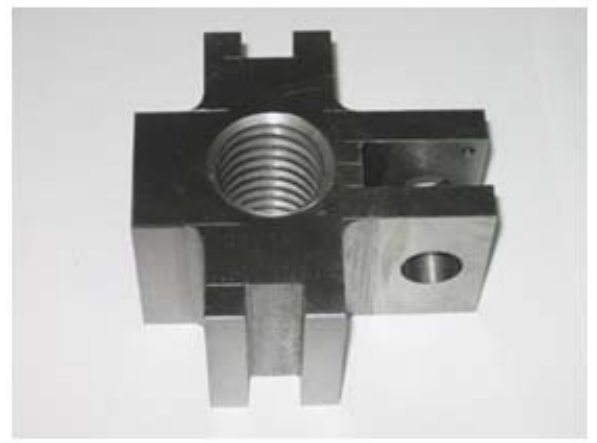

a)

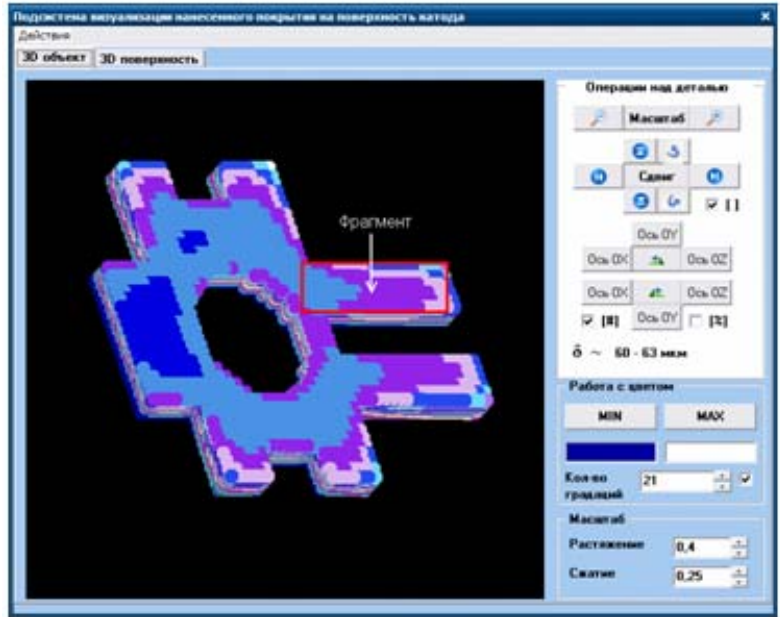

б)

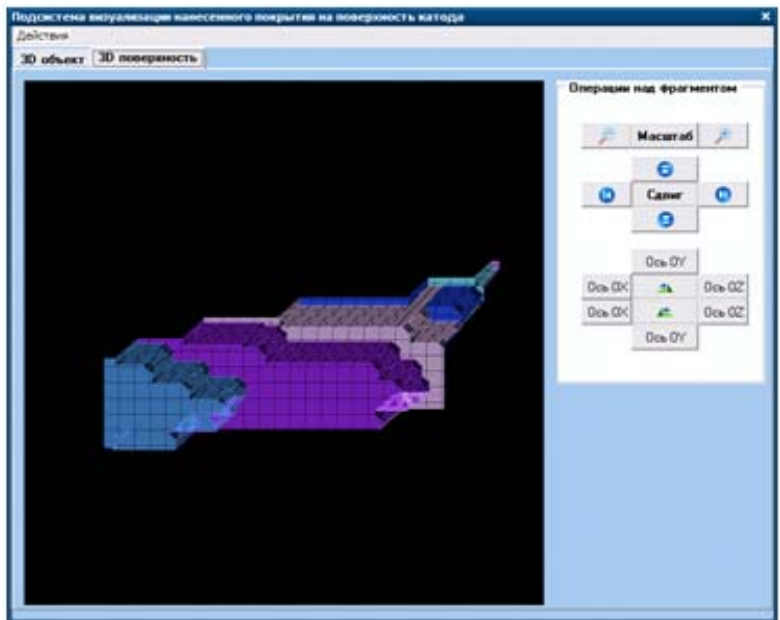

B)

Рисунок 1 - Деталь (а) и визуализированное расчетное распределение толщины покрытия по его поверхности (б) и фрагменту (в). 
Разработанное программное обеспечение обладает универсальностью и может использоваться не только в качестве подсистемы визуализации в автоматизированной системе управления гальваническими процессами с циклически включаемыми анодными секциями, но и в системах управления гальваническими процессами в ваннах со многими анодами [7, с. 522], токонепроводящим перфорированным экраном [8, с. 35], биполярными электродами [9, с. 328], источниками реверсивного [10, с. 407], импульсного [11, с. 1077] и асимметричного переменного тока $[12$, с. 31] и других, где является необходимым наглядная демонстрация распределения толщины покрытий на деталях, полученных в результате численных расчетов или экспериментальных измерений.

Работа выполнена при финансовой поддержке программы «У.М.Н.И.К.» Фонда содействия развитию малых форм предприятий в научно-технической сфере, гос.контракт №10741p/16955 от 01.08.2012г. на тему: «Разработка программного комплекса для системы управления гальванической ванной с циклическим включением анодных секций».

\section{References:}

1. Solovjev DS, Litovka JuV, Milovanov IV (2011) Optimizacija rezhimov raboty gal'vanicheskoj vanny s ciklicheskim vkljucheniem anodnyh sekcij. Radiotehnika, No. 5, pp. $71-77$.

2. Solovjev DS, Litovka JuV (2012) Proverka adekvatnosti matematicheskoj modeli raspredelenija gal'vanicheskogo pokrytija na detali $\mathrm{v}$ mnogoanodnoj vanne. Vestnik Tambovskogo gosudarstvennogo tehnicheskogo universiteta, vol. 18, No. 1, pp. $128-135$.

3. Solovjev DS, Litovka JuV (2012) Avtomatizirovannaja sistema upravlenija gal'vanicheskimi processami v mnogoanodnoj jelektrohimicheskoj vanne. Promyshlennye ASU i kontrollery, No. 7, pp. $15-22$.

4. Solovjev DS (2012) Svidetel'stvo o gosudarstvennoj registracii programmy dlja ECM v FIPS №2012615096 «Programma vizualizacii raspredelenija gal'vanicheskogo pokrytija po poverhnosti detali». Zaregistrirovano v reestre programm dlja ECM 07.06.2012.

5. Rejnbou V (2003) Komp'juternaja grafika. Jenciklopedija. SPb: Izdatel'skij dom «Piter», pp.768.

6. Golovanov NN (2002) Geometricheskoe modelirovanie. Moscow: Izdatel'stvo «Fizmatlit», pp. 472.

7. Helle HPE, Beek GHM, Ligtelijn JTh (1981) Numerical determination of potential distribution and current densities in multi-electrode systems. Corrosion, vol. 37(9), pp. $522-$ 530.

8. Protalinskij OM, Litovka JuV, Pashkevich AA (2009) Sistema optimal'nogo upravlenija gal'vanicheskoj vannoj s tokoneprovodjashhim jekranom. Datchiki i sistemy, No. 5, pp. $35-$ 36.

9. Litovka JuV, Miheev VV (2006) Chislennyj raschjot jelektricheskogo polja v gal'vanicheskoj vanne s bipoljarnymi jelektrodami. Teoreticheskie osnovy himicheskoj tehnologii, vol. 40, No.3, pp. 328 - 334.

10. Ismail MI (1979) Periodic reverse current electroplating and surface finishing. Journal of Applied Electrochemistry, May 1979, vol. 9, pp. $407-410$.

11. Zemanová M, Krivosudská M, Chovancová M, Jorík V (2011) Pulse current electrodeposition and corrosion properties of $\mathrm{Ni}-\mathrm{W}$ alloy coatings. Journal of Applied Electrochemistry, September 2011, vol. 41, pp. 1077 - 1085.

12. Dini JW, Johnson HR (1980) The properties of gold deposits produced by DC, pulse and asymmetric AC plating. Gold Bulletin, March 1980, vol. 13, pp. $31-34$. 